\title{
Advanced control with a Cooper-pair box: stimulated Raman adiabatic passage and Fock-state generation in a nanomechanical resonator
}

\author{
Jens Siewert, ${ }^{1,2}$ Tobias Brandes, ${ }^{3}$ and Giuseppe Falci ${ }^{1}$ \\ ${ }^{1}$ MATIS-INFM, Consiglio Nazionale delle Ricerche, \\ and Dipartimento di Metodologie Fisiche e Chimiche per l'Ingegneria, Universita di Catania, I-95125 Catania, Italy \\ ${ }^{2}$ Institut für Theoretische Physik, Universität Regensburg, D-93040 Regensburg, Germany \\ ${ }^{3}$ Department of Physics, The University of Manchester, Manchester, United Kingdom
}

(Dated: June 24, 2018)

\begin{abstract}
The rapid experimental progress in the field of superconducting nanocircuits gives rise to an increasing quest for advanced quantum-control techniques for these macroscopically coherent systems. Here we demonstrate theoretically that stimulated Raman adiabatic passage (STIRAP) should be possible with the quantronium setup of a Cooper-pair box. The scheme appears to be robust against decoherence and should be realizable even with the existing technology. As an application we present a method to generate single-phonon states of a nanomechnical resonator by vacuum-stimulated adiabatic passage with the superconducting nanocircuit coupled to the resonator.
\end{abstract}

PACS numbers: 32.80.Qk, 73.23.-b, 73.40.Gk

One of the most fascinating experimental breakthroughs of the recent past is the observation of quantumcoherent dynamics in superconducting nanocircuits. It includes circuits exhibiting the dynamics of single 'artificial atoms' [1, 2, 3], two coupled artificial atoms [4, 5] and artificial atoms coupled to electromagnetic resonators [6, 7]. This development opens new perspectives to study quantum phenomena in solid-state devices that traditionally have been part of nuclear magnetic resonance, quantum optics, and cavity quantum electrodynamics. There exist already a large number of theoretical proposals for such studies such as, e.g., the detection of geometric phases [8], the preparation of Schrödinger cat states in electrical and nanomechanical resonators 9, 10], cooling techniques [1], an analogue of electromagnetically induced transparency [12], and adiabatic passage in superconducting nanocircuits $13,14,15$.

One of the challenges is the preparation of Fock states in a resonator coupled to a superconducting nanocircuit. In quantum optics, the analogous problem has been solved both theoretically and experimentally 16, 17]. The idea is to apply adiabatic passage to the dark state of a three-level atom. Instead of driving the transition with two classical fields as in conventional STIRAP [18], one of the external fields is replaced by the quantum field of the cavity. While the atom undergoes the transition, a single photon is emitted into the cavity.

In the following we will demonstrate the application of this scheme to a Cooper-pair box operated as in the experiments by Vion et al. [2] (the so-called quantronium device) coupled to a nanomechanical resonator. To this end, we need to make sure that adiabatic passage in a three-level system using classical fields can be realized with the quantronium setup of a Cooper-pair box. This circuit is appropriate for the substitution of one of the classical driving fields by the quantum field of the nanomechanical resonator without changing the functionality of the Cooper-pair box. Coupling the resonator to the nanocircuit and verification of the vacuum-assisted adiabatic passage completes the analogue of the atomcavity system in Refs. 16, 17]. We will discuss also the effects of decoherence on the scheme in a real experiment.

We remark that, in principle, this program can be carried out for different regimes and setups of superconducting nanocircuits. (An alternative realization is a flux qubit coupled to an electrical resonator studied by Mariantoni et al. 19].) We have chosen the quantronium as, on the one hand, it is very much analogous to the atom-laser system in quantum optics and, on the other hand, it is a rather thoroughly studied system with respect to its decoherence properties.

Quantronium in a three-level STIRAP scheme. Adiabatic passage in three-level atoms is commonly realized with the STIRAP technique which is based on a $\Lambda$ configuration of two hyperfine ground states $|g\rangle$ and $|u\rangle$ coupled to an excited state $|e\rangle$ (with energies $E_{\mathrm{g}}, E_{\mathrm{u}}, E_{\mathrm{e}}$ ) by classical laser fields $A_{\mathrm{g}} \cos \omega_{\mathrm{g}} t, A_{\mathrm{u}} \cos \omega_{\mathrm{u}} t[18,20]$. In the frame rotating with the frequencies of the driving fields $\omega_{\mathrm{g}}, \omega_{\mathrm{u}}$ the Hamiltonian reads (applying the rotating-wave approximation)

$$
H_{\text {rot.f. }}=\Delta|e\rangle\langle e|+\frac{1}{2}\left(A_{\mathrm{u}}|e\rangle\left\langle u\left|+A_{\mathrm{g}}\right| e\right\rangle\langle g|+\text { h.c. }\right)
$$

with the detuning $\Delta=E_{\mathrm{e}}-E_{\mathrm{g}}-\omega_{\mathrm{g}}=E_{\mathrm{e}}-E_{\mathrm{u}}-\omega_{\mathrm{u}}$. This Hamiltonian has a so-called dark state

$$
|D\rangle=\frac{1}{\sqrt{\left|A_{\mathrm{u}}\right|^{2}+\left|A_{\mathrm{g}}\right|^{2}}}\left(A_{\mathrm{g}}|u\rangle-A_{\mathrm{u}}|g\rangle\right) .
$$

From Eq. (2) it can be seen that by slowly varying the coupling strengths $A_{\mathrm{u}}, A_{\mathrm{g}}$ the dark state can be rotated in the two-dimensional subspace spanned by $|u\rangle$ and $|g\rangle$. For the so-called counterintuitive scheme, the system is prepared in the state $|g\rangle$ with the couplings $A_{\mathrm{g}}=0$ and $A_{\mathrm{u}} \neq 0$. By slowly switching $A_{\mathrm{u}}$ off while $A_{\mathrm{g}}$ is switched on, the population can be transferred from state $|g\rangle$ to state $|u\rangle$. Adiabaticity requires $\left|\dot{A}_{j} / A_{j}\right|<\omega_{j}(j=u, g)$. 

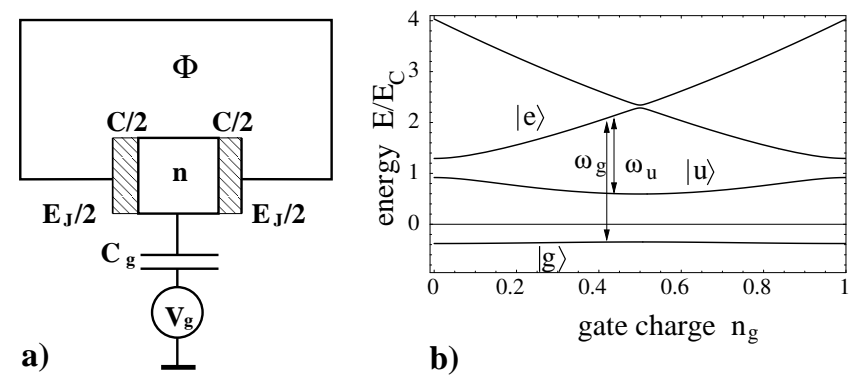

FIG. 1: a) In the quantronium setup, a superconducting island of total capacitance $C$ is coupled to a superconducting lead via two Josephson junctions. The gate voltage controls the offset charge of the island via the gate capacitance $C_{\mathrm{g}} \ll$ $C$. The magnetic flux $\Phi$ represents another control parameter for the setup (here we choose $\Phi=0$ ). b) The lowest four energy levels of the quantronium with $E_{\mathrm{C}}=E_{\mathrm{J}}$ as a function of gate charge. At the working point $n_{\mathrm{g} 0}$ the three lowest levels can be used as a $\Lambda$ scheme $|g\rangle,|u\rangle,|e\rangle$ with resonance frequencies $\hbar \omega_{\mathrm{g}}=E_{\mathrm{e}}-E_{\mathrm{g}}$ and $\hbar \omega_{\mathrm{u}}=E_{\mathrm{e}}-E_{\mathrm{u}}$.

In order to realize adiabatic population transfer with the quantronium setup (see Fig. 1a) consider the corresponding Hamiltonian in the basis of the charge states $|n\rangle$

$$
H=\sum_{n} E_{\mathrm{C}}\left(n-n_{g}(t)\right)^{2}|n\rangle\langle n|+\frac{E_{\mathrm{J}}}{2}(|n\rangle\langle n+1|+\text { h.c. })
$$

with the charging energy $E_{\mathrm{C}}=(2 e)^{2} /(2 C)$ (where $C$ is the total capacitance of the island and $(2 e)$ the charge of a Cooper pair) and the Josephson coupling energy $E_{\mathrm{J}}$. For the time being we assume $E_{\mathrm{C}}=E_{\mathrm{J}}$. The offset charge $n_{\mathrm{g}}=C_{\mathrm{g}} V_{\mathrm{g}} /(2 e)$ can be tuned with the gate voltage $V_{\mathrm{g}}$. In the quantronium setup, the gate voltage (and hence the gate charge) has a d.c. part $n_{\mathrm{g} 0}$ and an a.c. part $n_{\mathrm{g}}^{\mathrm{ac}}=A \cos \omega t$ with a small amplitude $|A| \ll 1 / 2$.

The STIRAP operation can be carried out between the three lowest energy levels (see Fig. 1b). For the working point $n_{\mathrm{g} 0}$ values such as $n_{\mathrm{g} 0}=1 / 2$ are preferable that lead to low decoherence rates. However, at symmetry points small level spacings and selection rules may impede the operation of the scheme [15]. Therefore the working point needs to be chosen away from such points, e.g., at $n_{\mathrm{g} 0}=0.45$. If two resonant frequencies $\omega_{\mathrm{g}}, \omega_{\mathrm{u}}$ are applied to the gate (see Fig. 1b), it is possible to adiabatically transfer the population from the ground state $|g\rangle$ to the first excited state $|u\rangle$. It is interesting to note that the microwave field couples diagonally to the charge states (as opposed to the dipole coupling in the threelevel atom case). Nevertheless, an effective Hamiltonian as in Eq. (11) is obtained as only those off-diagonal matrix elements in the eigenbasis of the driven Hamiltonian are important that couple two states resonantly 21.

In Fig. 2b-d (solid lines) we show the numerical solution of the Schrödinger equation for the Hamiltonian Eq. (3) with a gate charge $n_{\mathrm{g}}(t)=n_{\mathrm{g} 0}+A_{\mathrm{g}}(t) \cos \omega_{\mathrm{g}} t+$ $A_{\mathrm{u}}(t) \cos \omega_{\mathrm{u}} t$ (zero detuning, $n_{\mathrm{g} 0}=0.45$ ). Initially the system is prepared in the state $|g\rangle$. Then, two Gaussian- shaped microwave pulses are applied (cf. Fig. 2a). We observe that a population transfer to state $|u\rangle$ of nearly unit efficiency can be achieved. The state $|e\rangle$ practically does not get populated during the STIRAP procedure (cf. Fig. 2d). Note that there are many parameters that may be used to optimize the efficiency such as duration, delay, relative height and over-all shape of the pulses, the detunings etc. [18].

Effects of decoherence. The functionality of solid-state quantum-coherent devices is rather sensitive to various (often device-dependent) sources of decoherence. In the quantronium, high-frequency noise that is mainly responsible for unwanted transitions, coexists with lowfrequency noise which mainly affects calibration of the device and determines power-law reduction of the amplitude of the signal [22, 23].

A detailed analysis of decoherence in the STIRAP protocol due to a solid-state environment is beyond the scope of this work. Here we only estimate the feasibility of the protocol and argue that the main processes determining decoherence do not involve the level $|e\rangle$. These processes have been well characterized and, as a matter of fact, do not prevent very long decoherence times in the quantronium. We start our analysis from the quantum-optical master equation $\dot{\rho}=\frac{i}{\hbar}\left[\rho, H^{\prime}\right]-\Gamma \rho$ where $\rho$ is the density matrix and $H^{\prime}$ is the Hamiltonian (3) in the rotating frame 24]. At low temperature the dissipator $\Gamma \rho$ includes spontaneous decay rates of the excited states $|e\rangle,|u\rangle$ as well as environment-assisted absorption between eigenstates in the presence of the laser coupling. In quantumoptical systems the rate $\gamma_{\mathrm{u} \rightarrow \mathrm{g}}$ vanishes and the remaining

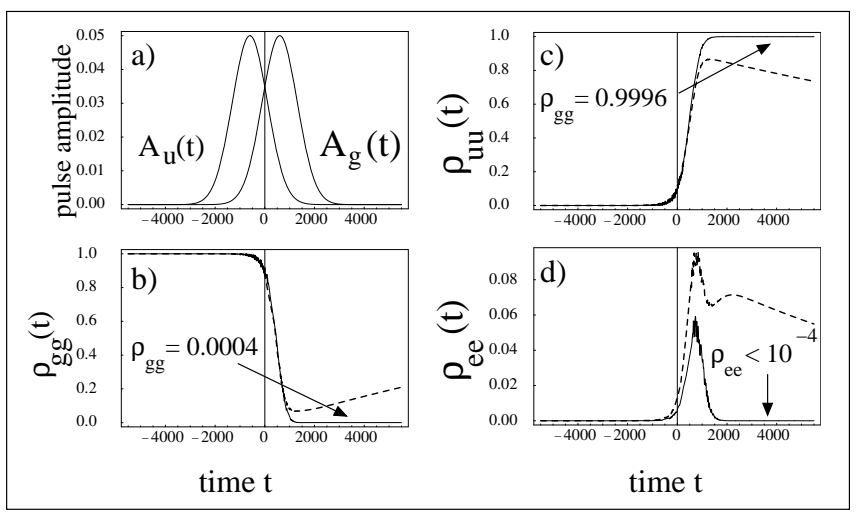

FIG. 2: Population transfer by STIRAP in the quantronium setup $\left(n_{\mathrm{g} 0}=0.45\right)$. a) Gaussian pulses are applied in the counterintuitive scheme. The maximum gate charge of the microwave fields are $\max \left(A_{\mathrm{u}}(t), A_{\mathrm{g}}(t)\right)=0.05$. For a charging energy of $E_{\mathrm{C}}=50 \mu \mathrm{eV}$ the time unit corresponds to about $1.3 \times 10^{-11} \mathrm{~s}$. b) $-\mathrm{d}$ ) Time evolution of the populations $\rho_{\mathrm{gg}}$, $\rho_{\text {uu }}, \rho_{\text {ee }}$ without decoherence (solid lines) and with decoherence (dashed lines). The arrows denote the final populations in the ideal case (no decoherence). For the calculations with decoherence we have used the decay rate $\gamma_{\mathrm{u}}=4.4 \times 10^{-5}$ and the dephasing rate $\tilde{\gamma}=2.6 \times 10^{-4}$. The latter rate corresponds to a dephasing time of about $50 \mathrm{~ns}$. 
processes mainly act towards depopulating states while they are not populated, and therefore hardly affect the protocol. In contrast, STIRAP for the quantronium may be sensitive to the extra decay $|u\rangle \rightarrow|g\rangle$ involving the two low-lying states.

An estimate of the effect of decoherence is achieved by studying the master equation (written in the basis $\{|g\rangle,|u\rangle,|e\rangle\})$ with the dissipator

$$
(\Gamma \rho)_{i j}=\frac{\gamma_{i}+\gamma_{j}}{2} \rho_{i j}-\left(1-\delta_{i j}\right) \tilde{\gamma}-\delta_{i j} \sum_{k} \rho_{k k} \gamma_{k \rightarrow i}
$$

where $\gamma_{i}=\sum_{k \neq i} \gamma_{\mathrm{i} \rightarrow \mathrm{k}}$. The dissipator is taken timeindependent (which overestimates decoherence) and includes all transitions as well as a dephasing rate $\tilde{\gamma}$ accounting phenomenologically for low-frequency noise. For the decay rate of the second excited state we assume $\gamma_{\mathrm{e}}=\gamma_{\mathrm{e} \rightarrow \mathrm{u}}+\gamma_{\mathrm{e} \rightarrow \mathrm{g}}=2 \gamma_{\mathrm{u}}$. In order to obtain a realistic estimate of decoherence effects, rates on the order of those observed in the experiments of Ref. 23] are used.

The dashed lines in Fig. 2b-d show results for the solution of the master equation with the dissipator (4). One recognizes immediately a remarkable robustness of the STIRAP procedure against decoherence. The main noticeable effects are the variation of populations during the waiting time after finishing the pulse sequence and a slightly increasing population of level $|e\rangle$.

Low-frequency noise is modeled more realistically as due to impurities which are static during each run of the protocol but may switch on a longer time scale, thus leading to statistically distributed level separations. Averaging determines defocusing of the signal. Fluctuations of $E_{\mathrm{e}}$ may be relatively large, but they represent equal detunings of both microwave fields and do not affect STIRAP. On the other hand, fluctuations of the separation between the two lowest eigenstates are potentially detrimental since they determine fluctuations of the difference of detunings. This leads to a reduced efficiency of population transfer which, however, may be improved by optimizing the parameters of the protocol.

Coupling the quantronium to a harmonic-oscillator mode. As we have demonstrated, STIRAP should be well within reach of present-day technology for superconducting nanocircuits. Therefore one might hope to apply this technique similarly as in quantum optics for the preparation of peculiar quantum states. One such application is the generation of Fock states in a cavity coupled to a three-level atom [16]. For this purpose, the Cooper-pair box needs to be coupled to a harmonic oscillator degree of freedom. The generic coupling Hamiltonian is $H_{\text {int }}=\lambda\left(a+a^{\dagger}\right)\left(n-n_{\mathrm{g}}\right)$. There are various ways to implement this Hamiltonian with electrical resonator circuits [25] and transmission lines [6], or with nanomechanical resonators [10, 11]. In the following we will explain that along these lines it is possible to generate Fock states in a nanomechanical oscillator.

The nanomechanical oscillator (mass $m$ ) is coupled capacitively to the Cooper-pair
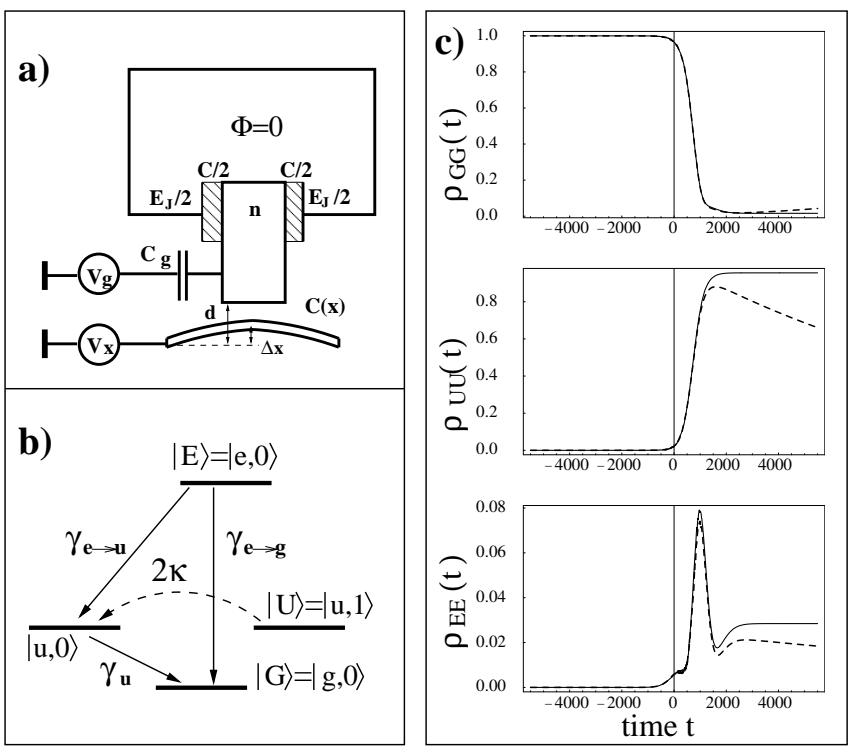

FIG. 3: a) Coupled system of quantronium and nanomechanical osciallator. b) The four relevant states of the STIRAP scheme for Fock state generation in the presence of decoherence. c) Level population for the quantroniumresonator setup in the presence of decoherence. Parameters are $n_{\mathrm{g} 0}(t)+n_{\mathrm{x}}(t)=0.03, \max \left(A_{\mathrm{g}}(t), \lambda(t)\right)=0.05, \gamma_{\mathrm{u}}=$ $4.4 \times 10^{-5}, \tilde{\gamma}=2.6 \times 10^{-4}, Q=5.0 \times 10^{3}$.

box [10, 11] via the position-dependent capacitance $C(x) \simeq C_{\mathrm{x}}+\Delta x(\mathrm{~d} C(x) / \mathrm{d} x)$, see Fig. 3a. Here $\Delta x$ denotes the oscillator displacement. The coupling can be tuned by the voltage $V_{\mathrm{x}}$. Assuming $\Delta x \ll d$ and taking into account only a single mode of the mechanical oscillator, the coupled quantronium-resonator system is described by the Hamiltonian [10, 11]

$$
\begin{aligned}
H= & H_{\mathrm{qu}}+H_{\mathrm{res}}+H_{\mathrm{int}}, \quad H_{\mathrm{res}}=\hbar \omega_{\mathrm{res}} a^{\dagger} a \\
H_{\mathrm{qu}}= & \sum_{n} E_{\mathrm{C}}\left(n-n_{\mathrm{g}}(t)-n_{\mathrm{x}}(t)\right)^{2}|n\rangle\langle n|+ \\
& \quad+\left(E_{\mathrm{J}} / 2\right)(|n\rangle\langle n+1|+\text { h.c. }) \\
H_{\mathrm{int}}= & E_{C} \frac{2 C_{\mathrm{x}} V_{\mathrm{x}}}{2 e} \frac{1}{d} \sqrt{\frac{\hbar}{2 m \omega_{\mathrm{res}}}}\left(a+a^{\dagger}\right)\left(n-n_{\mathrm{g}}\right) \\
\equiv & \lambda\left(a+a^{\dagger}\right)\left(n-n_{\mathrm{g}}\right)
\end{aligned}
$$

where $d$ is the distance of the resonator from the island and $a^{\dagger}, a$ denote the creation and annihilation operators for the nanomechanical oscillator. The total gate charge is now a sum of the box gate charge $n_{\mathrm{g}}(t)$ and $n_{\mathrm{x}}(t) \equiv$ $C_{\mathrm{x}} V_{\mathrm{x}}(t) /(2 e)$ where $n_{\mathrm{g}}(t)=n_{\mathrm{g} 0}(t)+A_{\mathrm{g}}(t) \cos \omega_{\mathrm{g}} t$. The composed system is described by the basis states $|j, N\rangle \equiv$ $|j\rangle \otimes|N\rangle$ with the (uncoupled) quantronium eigenstates $|j\rangle$ and the resonator Fock states $|N\rangle$. For the states relevant in our discussion we will use the notation $|G\rangle=$ $|g, 0\rangle,|U\rangle=|u, 1\rangle$, and $|E\rangle=|e, 0\rangle$.

We assume that it is possible to prepare the vacuum state $|G\rangle$, i.e., the oscillator frequency $\omega_{\text {res }}$ has to be sufficiently large compared to the temperature in the experiment (for a discussion of possible values in an experi- 
ment see below). The population transfer is performed from the initial state $|G\rangle$ via $|E\rangle$ to the state $|U\rangle$. As the "Stokes field" $A_{\mathrm{u}}(t)$ is replaced by the vacuum field of the cavity (which is coupled via the quantronium-resonator coupling parameter $\lambda(t)$ ), a single phonon is emitted into the resonator during the STIRAP operation. Again, the cavity field may trigger transitions between eigenstates of the setup although it has only terms diagonal in the charge basis due to mixing of charge states by the Josephson coupling.

As mentioned above, $k_{\mathrm{B}} T<\hbar \omega_{\text {res }}$ is required. With typical temperatures of $k_{\mathrm{B}} T \lesssim 30 \mathrm{mK}$, oscillator frequencies above $\omega_{\text {res }}>2 \pi \times 1 \mathrm{GHz}$ are necessary (which is at the limit of present-day technology [26]). Note also that the oscillator frequency needs to be resonant with the quantronium transition $u \rightarrow e$. With a charging energy of $E_{\mathrm{C}} \approx 2 E_{\mathrm{J}} \sim 35 \mu \mathrm{eV}$ and $n_{\mathrm{g} 0} \sim 0.03$ it is possible to have $\omega_{\text {res }} \sim 2 \pi \times 1.5 \mathrm{GHz}$. With these parameters one may hope to achieve similar decoherence effects as in the experiments of Refs. 2, 23] and, at the same time, to generate the appropriate level spacings. Assuming the same decoherence rates as in the STIRAP process with classical microwave fields (Fig. 2) and taking into account a finite quality factor of the nanomechanical resonator $Q=\omega_{\text {res }} /(2 \kappa)=5.0 \times 10^{3}$ we can numerically evaluate the time evolution of the coupled system. Note that for this calculation it is necessary to take into account also the state $|u, 0\rangle$ which is not part of the STIRAP scheme (see Fig. 3b) but contributes to reduce coherence of the population transfer. It can be seen that a highly efficient transfer of the system to the state $|U\rangle$ should be feasible (cf. Fig. 3c).

As to the detection, it would be desirable to directly measure the state of the oscillator. However, it may be easier to probe the state $|U\rangle$ via a measurement of the quantronium eigenstate. Either, one probes merely the final state $|u\rangle$. Alternatively, the system can be viewed as a realization of the Jaynes-Cummings model [20] and one may try to detect Rabi oscillations between the states
$|U\rangle$ and $|E\rangle$ induced by the cavity field. To this end, the resonator-box coupling $\lambda(t)$ needs to be set to the appropriate value that facilitates the observation of such Rabi oscillations (while $A_{g} \equiv 0$ ). Note that for this type of detection high-quality resonators are required, and it is necessary to distinguish between the quantronium eigenstates $|u\rangle$ and $|e\rangle$.

The procedure described here is not limited (at least in theory) to the generation of single-phonon states of the resonator [16]. The final state $|U\rangle=|u, 1\rangle$ of the protocol described so far may be changed (via a $\pi$ pulse in the quantronium with vanishing resonator-box coupling) into $|g, 1\rangle$. This state may serve as the initial state for another STIRAP transfer $|g, 1\rangle \rightarrow(|e, 1\rangle) \rightarrow|u, 2\rangle$, etc.

It is an important advantage of the STIRAP protocol for its realization in solid-state devices that the efficiency does not depend sensitively on the absolute values of the couplings during the procedure. This makes it robust against fluctuations in the environment. Another advantage is its versatility. For example, instead of changing the amplitudes of the driving fields it is possible to change the driving frequencies [18]. This may be an option for a Cooper-pair box coupled to an electrical resonator such as in Ref. [25] where it is easier to change the resonator frequency than the capacitive coupling. Interestingly, the protocol to generate Fock states can even be modified such that it suffices to switch the couplings from $A_{\mathrm{g}}(t) \ll \lambda(t)$ at time $t$ to $A_{\mathrm{g}}\left(t^{\prime}\right) \gg \lambda\left(t^{\prime}\right)$ at $t^{\prime}>t$ (see Ref. 24]). That is, a single phonon (or photon) can be generated and emitted from the cavity with an 'always-on' cavity coupling. This may be interesting for setups where both coupling and resonator frequency are fixed such as in Ref. [6].

Acknowledgments This work has been supported financially by SFB 631 of the DFG. JS would like to thank P. Schlagheck for pointing out to him Ref. [17] and D. Esteve for stimulating comments. Illuminating discussions with A. Kuhn and M. Storcz are gratefully acknowledged.
[1] Y. Nakamura, Yu. Pashkin, and J.S. Tsai, Nature 398, 786 (1999).

[2] D. Vion, A. Aassime, A. Cottet, P. Joyez, H. Pothier, C. Urbina, D. Esteve, and M.H. Devoret, Science 296, 886 (2002).

[3] I. Chiorescu, Y. Nakamura, C.J.P.M. Harmans, and J.E. Mooij, Science 299, 1869 (2003).

[4] T. Yamamoto, Yu.A. Pashkin, O. Astafiev, Y. Nakamura, and J.S. Tsai, Nature 425, 941 (2003).

[5] J.B. Majer, F.G. Paauw, A.C.J. ter Haar, C.J.P.M. Harmans, and J.E. Mooij, Phys. Rev. Lett. 94, 090501 (2005).

[6] A. Wallraff, D.I. Schuster, A. Blais, L. Frunzio, R.S. Huang, J. Majer, S. Kumar, S.M. Girvin, and R.J. Schoelkopf, Nature 431, 162 (2004).

[7] I. Chiorescu, P. Bertet, K. Semba, Y. Nakamura, C.J.P.M. Harmans, and J.E. Mooij, Nature 431, 159
(2004).

[8] G. Falci, R. Fazio, G.M. Palma, J. Siewert, and V. Vedral, Nature 407, 355 (2000); L. Faoro, J. Siewert, and R. Fazio, Phys. Rev. Lett. 90, 028301 (2003); M. Cholascinski, Phys. Rev. B 69, 134516 (2004).

[9] F. Marquardt and C. Bruder, Phys. Rev. B 63, 054514 (2001).

[10] A. Armour, M. Blencowe, and K.C. Schwab, Phys. Rev. Lett. 88, 148301 (2002).

[11] I. Martin, A. Shnirman, L. Tian, and P. Zoller, Phys. Rev. B 69, 125339 (2004); P. Zhang, Y.D. Wang, and C.P. Sun, Phys. Rev. Lett. 95, 097204 (2005).

[12] K.V.R.M. Murali, Z. Dutton, W.D. Oliver, D.S. Crankshaw, and T. Orlando, Phys. Rev. Lett. 93, 087003 (2004).

[13] M.H.S. Amin, A.Yu. Smirnov, and A. Maassen v.d. Brink, Phys. Rev. B 67, 100508(R) (2003). 
[14] J. Siewert and T. Brandes, Adv. Solid State Phys. 44, 181 (2004).

[15] Y.-X. Liu, J.Q. You, L.F. Wei, C.P. Sun, and F. Nori, Phys. Rev. Lett. 95, 087001 (2005).

[16] A.S. Parkins, P. Marte, P. Zoller, H.J. Kimble, Phys. Rev. Lett. 71, 3095 (1993).

[17] M. Henrich, T. Legero, A. Kuhn, and G. Rempe, Phys. Rev. Lett. 85, 4872 (2000).

[18] K. Bergmann, H. Theuer, and B.W. Shore, Rev. Mod. Phys. 70, 1003 (1998); N.V. Vitanov, T. Halfmann, B.W. Shore, and K. Bergmann, Annu. Rev. Phys. Chem. 52, 763 (2001).

[19] M. Mariantoni, M.J. Storcz et al., submitted (2005).

[20] M.O. Scully and M.S. Zubairy: Quantum Optics (Cambridge Univ. Press, Cambridge 1997).
[21] M.A. Kmetic, R.A. Thuraisingham, and W.J. Meath, Phys. Rev. A 33, 1688 (1986).

[22] E. Paladino, L. Faoro, G. Falci, and R. Fazio, Phys. Rev. Lett. 88, 228304 (2002); G. Falci, A. D'Arrigo, A. Mastellone, and E. Paladino , Phys. Rev. Lett. 94, 167002 (2005).

[23] G. Ithier, E. Collin, P. Joyez, P.J. Meeson, D. Vion et al., eprint condmat/0508588 (2005).

[24] A. Kuhn, M. Hennrich, T. Bondo, and G. Rempe, Appl. Phys. B 69, 373 (1999).

[25] F. Plastina and G. Falci, Phys. Rev. B 67, 224514 (2003).

[26] X.M.H. Huang, C.A. Zorman, M. Mehregany, and M. Roukes, Nature 421, 496 (2003). 